\title{
The BDNF Val66Met Polymorphism is a Relevant, But not Determinant, Risk Factor in the Etiology of Neuropsychiatric Disorders - Current Advances in Human Studies: A Systematic Review
}

\author{
Gilmara Gomes de Assis ${ }^{\mathrm{a}, \mathrm{b}, *}$ and Jay R. Hoffman ${ }^{\mathrm{c}}$ \\ ${ }^{a}$ Laboratory of Endocrinology, Brain Institute, Federal University of Rio Grande do Norte, Brazil \\ ${ }^{\mathrm{b}}$ Gdansk University of Physical Education and Sports, Faculty of Physical Education, Gdansk, Poland \\ ${ }^{\mathrm{c}}$ Department of Physical Therapy, Ariel University, Ariel, Israel
}

\author{
Accepted 15 February 2022 \\ Pre-press 7 March 2022 \\ Published 20 December 2022
}

\begin{abstract}
Brain-derived neurotrophic factor (BDNF) is the brain's most-produced neurotrophin during the lifespan, essentially involved in multiple mechanisms of nervous system development and function. The production/release of BDNF requires multi-stage processing that appears to be regulated at various stages in which the presence of a polymorphism "Val66Met" can exert a critical influence.

Aim: To synthesize the knowledge on the BDNF Val66Met polymorphism on intracellular processing and function of BDNF. Methods: We performed a systematic review and collected all available studies on the post-translation processes of BDNF, regarding the Val66Met polymorphism. Searches were performed up to 21st March 2021.

Results: Out of 129 eligible papers, 18 studies addressed or had findings relating to BDNF post-translation processes and were included in this review.

Discussion: Compilation of experimental findings reveals that the Val66Met polymorphism affects BDNF function by slightly altering the processing, distribution, and regulated release of BDNF. Regarding the critical role of pro-BDNF as a pro-apoptotic factor, such alteration might represent a risk for the development of neuropsychiatric disorders.
\end{abstract}

Keywords: Val66Met polymorphism, pro-BDNF cleavage, Post-translation, BDNF secretion, Brain development, Neuroplasticity

\section{INTRODUCTION}

Brain-derived neurotrophic factor (BDNF) is the most studied neurotrophin that has an essential role in the development and function of the central nervous

\footnotetext{
*Correspondence to: Gilmara Gomes de Assis, Gdansk University of Physical Education and Sports, Faculty of Physical Education, Gdansk, Poland; E-mail: gilmara.gomesdeassis@awf.gda.pl; ORCID: 0000-0002-7198-3601.
}

system (CNS) [1, 2]. Mainly expressed in neurons and neural tissue, the pleiotropic roles of BDNF include neural stem cell survival and differentiation; axon-dendrite differentiation, growth and guidance; synapse formation and maturation; and survival of differentiated neurons [3-6].

The gene $B D N F$ primarily transcripts a pre-proBDNF mRNA strain which leaves the nucleus as pro-BDNF mRNA that undergoes translation, dimerization and cleavage modifications while trafficking 
through the endoplasmic reticulum (ER) and transGolgi network (TGN), before release as the mature BDNF. The complexity of BDNF processing before release involves both independent and integrated steps that are regulated at multiple stages, and has been well described by several investigations [7-9]. Release of BDNF from neurons occurs both in a regulated (in response to depolarization) and a constitutive (in a steady stream) manner $[10,11]$.

BDNF signaling, through its high affinity tropomyosin receptor kinase B $(\operatorname{TrkB})$, initiates several pro-growth and survival biochemical cascades within neural cells. Whereas the pro-BDNF precursor form is also an active factor which binds with lowaffinity to the common p75NTR receptor to induce pro-apoptosis pathways [12]. So that, regulation of the pro-BDNF to BDNF cleavage is critical during the brain's development, as well as in neuron-specific activities such as long-term synaptic formation and/or depression. Impairment in any of these mechanisms can result in errors of neuronal circuit formations and/or activity $[13,14]$.

A well-studied polymorphism situated on the p13 region of chromosome 11 referring to the substitution of an adenine (A) by a guanine (G) base in the $B D N F$ gene causes a shift from valine to methionine in position 66 of the pro-BDNF isoform - specifically at the pro-domain region [15]. This polymorphism has been identified in 20-30\% of the genotyped subpopulations afflicted with various neurological disorders [16]. Although, the $B D N F$ Val66Met polymorphism alone is not a determinant factor for the diagnosis of neuropathology, several studies have suggested that the $B D N F$ Val66Met polymorphism has a role in the etiology of a variety of neuropsychiatric disorders, such as depression, schizophrenia, and bipolar disorder [17-23].

In the first in situ study identifying a difference in the allele-specific expression of the BDNF containing the Val66met polymorphism, a decrease in the expression levels of $B D N F$ was shown in individuals heterogenous for the Val66met polymorphism, which is not detected in circulating blood [24]. This is consistent with the available clinical studies to date that have been unable to demonstrate a relationship between the presence of the Val66met polymorphism and alterations in circulating BDNF concentrations [25-28]. The Val66met polymorphism is found more frequently in a small segment of the population that are undergoing stress-related disorders, and is associated with a poor treatment response [29-31].
Thus, to provide further insight on whether the Val66Met polymorphism is a risk factor for the developing and/or aggravation of a spectrum of neuropsychiatric related disorders, we synthetized the available scientific knowledge on the post-translational processes involved in BDNF release/secretion addressing the Val66Met polymorphism in vivo and in vitro.

\section{METHODS}

To address the available scientific literature on the possible implications of the human $B D N F$ Val66Met polymorphism on the intracellular processing and production of BDNF, we performed a systematic review using an analog strategy to the PICO strategy for clinical studies as follows: P - Experimental models in vivo/in vitro; I - Cells, tissue and animals transfected with the human $B D N F$ Val66Met polymorphism; C - Non-polymorphic BDNF; O Post- transcriptional processing and release/secretion mechanisms. The following combinations of the terms identified by the Medical Subject Headings (Mesh terms) were applied to the Advanced search in the Pubmed platform: [(Brain-derived neurotrophic factor precursor OR pro-BDNF) AND (Secretory rate $\mathbf{O R}$ secretory pathway $\mathbf{O R}$ Secretory vesicles OR Protein processing OR Post-translational process)] and [(Brain-derived neurotrophic factor $\mathbf{O R}$ BDNF) AND (Secretory rate OR secretory pathway OR Secretory vesicles OR Protein processing OR Post-translational process)]. All the papers retrieved with the search were screened by two independent researchers for the specific inclusion criteria of: studies addressing the human $B D N F$ Val66Met polymorphism in human or animal models, in vivo or in vitro. Reviews and studies with non-biological models or not presenting data of the $B D N F$ Val66Met polymorphism were excluded from results. The number of studies excluded with reasons are present in Fig. 1. Eighteen studies were included for discussion. Studies main findings were described in the Results section. Studies methods and data summary were displayed in Table 1. The searches were performed from September 9th, 2020 to March 21st, 2021. Mendeley desktop 1.19.4 was used to select the files.

\section{RESULTS}

There has only been one study to date that has addressed BDNF production (expression and secretion) and the $B D N F$ Val66Met polymorphism in 


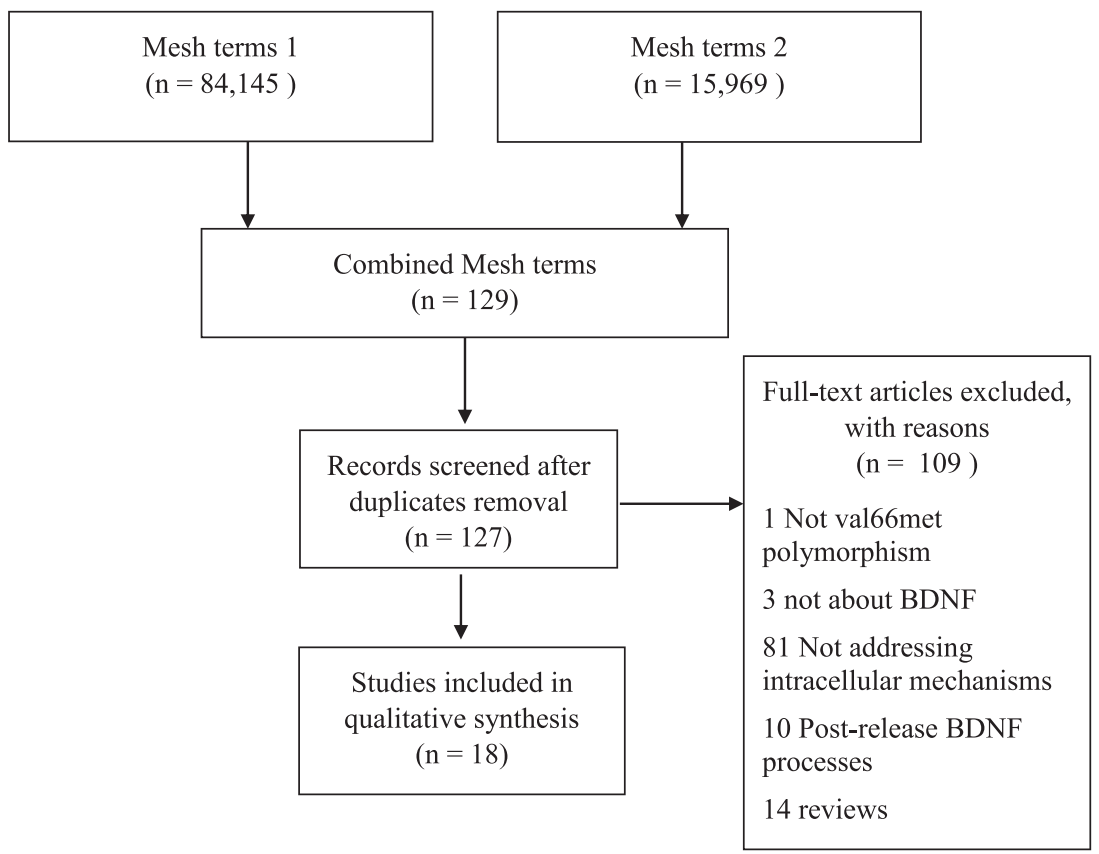

Fig. 1. PubMed search diagram.

humans [24]. Considering that this study was very recent, all the experimental studies that helped synthesize the knowledge on the $B D N F$ polymorphism impact on the proteins' production will follow a temporal order. Describing the post-translational mechanisms of $B D N F$ transcript products, Seidaha and colleagues [32] used a vaccine virus expression system in activity-deficient cells and demonstrated that endoproteases furin, PACE4, and PC5/6-B effectively process pro-BDNF in different cell types. While exploring how neurotrophins are sorted into secretory systems, Heymach and colleagues [33] showed that all members of the neurotrophin family are released via a regulated secretory pathway, and that their signal(s) for sorting lie within the neurotrophins moiety structure. Subsequently, Mowla et al. [34] was able to identify that neurotrophin sorting might differ from each other, by using the independent criteria of retention or release from cells after pulse-chase labeling, depolarization dependent release, and immunocytochemical localization of both nerve growth factor (NGF) and BDNF into recombinant virus-infected neuronal cells. Results showed that pro-BDNF is preferentially sorted into a regulated secretory pathway, while the bulk of newly synthesized NGF is sorted into the constitutive pathway. It was also revealed that pro-BDNF is not as efficiently cleaved by furin in TGN as is the
pro-NGF. These findings suggested that BDNF appears to be sorted into either a constitutive or regulated secretory pathway dependent on the efficiency of enzymatic cleavage into TGN. An additional investigation by Mowla and colleagues [35] described the $32-\mathrm{kDa}$ pro-BDNF isoform with $\mathrm{N}$-glycosylation in the pro-domain site. They reported that pro-BDNF undergoes an $\mathrm{N}$-terminal cleavage within the TGN and/or immature secretory vesicles to generate the mature BDNF form $(14 \mathrm{kDa})$. A $28-\mathrm{kDa}$ protein residue was also identified in the ER after the $\mathrm{N}$ terminal cleavage without a noticeable effect on BDNF production.

The first experiment to demonstrate that hippocampal neurons transfected by constructs encoding Val66- or Met66-BDNF to effect activity-dependent secretion of BDNF was performed by Egan et al. [36]. Their study showed a lower depolarization induced BDNF release, with no effects on the constitutive secretion system in Met66BDNF transfected neurons. In addition, they reported that Met66 containing pro-BDNF isoforms failed to localize to secretory granules or synaptic terminals in these cells, implying a Val66Met influence on the intracellular trafficking of BDNF. Subsequently, in vitro studies using cortical neurons co-transfected with both Val66BDNF and Met66BDNF coding constructs verified the expression products and locations within cells Chen, 
Table 1

Data summary of studies included by criteria

\begin{tabular}{lll}
\hline$\#$ & Authors & Aims \\
\hline 1 & Seidah, N. G., et al., 1996. & $\begin{array}{l}\text { To define the enzymes responsible for the maturation of the } \\
\text { precursors of the human BDNF and neurotrophin-3 }\end{array}$ \\
2 & Heymach, J. V., et al., 1996. & $\begin{array}{l}\text { To understand how different neurotrophins (nerve growth } \\
\text { factor, BDNF, and neurotrophin-3) are sorted and secreted by } \\
\text { neurons }\end{array}$
\end{tabular}

3 Mowla, S. J., et al., 1999.

4 Mowla, S. J., et al., 2001

5 Egan, M. F., et al., 2003.

6 Chen, Z. Y., et al., 2004.

7 Wang, L. C., et al., 2004.

8 Wu, Y. J. J., et al., 2004.

9 Li, H., et al., 2005.

10 Salio, C., et al., 2007.

11 Jiang, X., et al., 2009.

12 Dieni, S., et al., 2012

13 Petoukhov, E., et al., 2013.

14 Anastasia, A., et al., 2013.

15 Eckenstaler, R., et al., 2016.

16 Aravamudan, B., et al., 2016.

17 Uegaki, K., et al., 2017.

18 de Assis et al., 2021
To determine whether (NGF and BDNF) sorting differences are intrinsic to the neurotrophins or reflect differences between cell types.

To examinate the biosynthesis and post-translational processing of the pro-BDNF

To investigate underlying molecular mechanisms of trafficking of val- and met-BDNF proteins.

To examinate the effect of co-expressed BDNF Met on BDNF Val intracellular trafficking and processing.

To investigate (i) whether BDNF is present in all three secretory granule types; (ii) whether BDNF coexists with POMC and a $\mathrm{MSH}$ and (iii) whether the amount of BDNF in one or more of the secretory granule types is related to the secretory activity

To examinate the subcellular localization and release characteristics of NGF, BDNF, and NT-3 in adenovirus-infected primary cortical neurons.

to assess the trafficking of monoamine transporter 2 and the potential for monoamine release by exocytosis

To examinate the subcellular localization of BDNF and coexisting peptides under normal conditions or after intrathecal infusion of NGF.

To describe how BDNF isoform levels were regulated by chronic drug use.

To demonstrate that BDNf and its pro peptide localize in large dense core vesicles in excitatory presynaptic terminals

To demonstrate that PGRN is colocalized with dense-core vesicle markers and is co-transported with BDNF within axons and dendrites

To show that the isolated pro-BDNF is detected in the hippocampus and that it can be secreted from neurons in an activity- dependent manner.

To show that calcium-dependent activator protein 1 plays a role in regulating the intragranular $\mathrm{pH}$ and exocitoses of BDNF-containing secretory granules.

To dissect intrinsic mechanisms regulating endogenous, as well as in- flammation-induced BDNF secretion in ASM of nonasth- matic vs. asthmatic conditions.

To investigated the influence of the common Val66Met $B D N F$ polymorphism on the interaction between $\mathrm{BDNF}$ and the pro-peptide.

The Val66 and Met66 Alleles-Specific Expression of BDNF in Human Muscle and Their Metabolic Responsivity.
Model/Method

Rodent PC1, PC2, PC5, and PC5/6-B

cells; Human furin and PACE4.

Rodent cDNA subclones.

COS-7 and PC12 transfected cells.

Transient transfections of AtT-20,

PC12, and MDCK cells.

Cultured hippocampal neurons

Cultured hippocampal neurons

Rodent hippocampal neurons

Rodent cortical neurons cultures

Xenopus laevis melanotrope cells

Rodent cortical neurons cultures

Rodent neuroendocrine PC12 cells

Rodents spinal cord and amygdala coronal sections

Human brain biopsies

Rodent brain hippocampus

Cultured hippocampal neurons

Nuclear magnetic resonance spectroscopy and circular dichroism in rodent neurons

Primary cortical astrocytes and cultured hippocampal neurons.

Human airway smooth muscle cells

Computational Modeling and Recombinant human $B D N F$

Muscle biopsies from 13 male ( $34 \pm 9$ yo) were analyzed before and after a VO2max test. Allele-specific BDNF mRNA concentrations were quantified by ddPCR in heterozygous and homozygous. et al. [37]. The product of Val66 and Met66 BDNF co-expression contained $\sim 70 \%$ of Val66Met proBDNF heterodimers compared to either Val/Val or Met/Met BDNF labeled homodimers. When individually expressed in the cells, the Met66 BDNF products, in comparison to Val66 BDNF products, were localized more in the cell body then in the dendrites or axons. Wu et al. [38] described the subcellular localization and release of BDNF in cortical neurons by using confocal microscopy. They 
reported that BDNF co-localized with all members of the neurotrophin family in the ER and Golgi apparatus within cell bodies and were distributed in the large dense-core vesicles (DCVs) within axons and dendrites of neurons. Their study demonstrated that BDNF release, triggered by depolarization via a regulated secretory pathway, can be packaged into shared vesicles. Similarly, Wang and colleagues [39] quantified the intracellular BDNF isoforms in different stages of the regulated secretory pathway. Using immunoelectron microscopy, they reported that BDNF-immunoreactivity increases along with the secretory granule maturation axis, from immature to mature secretory granules. The authors were able to show that, in opposition to large DCVs, immature and low-density secretory granules contain primarily pro-BDNF molecules, and that the high-density secretory granules represent the stage in which proBDNF is processed into mature BDNF. Subsequent investigations by both Li et al. [40] and Salio and colleagues [41] confirmed that BDNF is stored in DCVs, as well as in neuronal tissue of both the central and peripheral nervous systems. Moreover, BDNF is colocalized with the vesicular monoamine transporter 2 within the DCVs. Because the location of vesicular monoamine transporter 2 defines a population of secretory vesicles that mediate the activity-dependent somato-dendritic release of multiple retrograde signals, these findings help elucidate the mechanism of the somato-dendritic release of BDNF by neural cells.

Jiang and colleagues [42], examined hippocampal neurons transfected by constructs encoding Val66or Met66-BDNF differing in their signal peptide (pre-regions) - referred to as short BDNF, BDNF1 and BDNF2 variants. In basal conditions, the cells expressing each variant released the same amount of BDNF, regardless of the polymorphism. When depolarization occurred, a decrease was detected in the pattern of BDNF secretion for the short-BDNF Met66 compared to Val66, but not for BDNF1 or BDNF2, which turned out to be greater than those of short BDNF. Consistent with earlier observations, Dieni et al. [43] highlighted that BDNF and pro-BDNF are prominently distributed in the cell body and in axon terminals. They demonstrated that both BDNF and its cleaved pro-peptide are found in large DCVs located in presynaptic terminals of excitatory neurons at roughly equimolar ratios, while the lack of BDNF expression in dendritic spines suggested an anterograde role of BDNF in neurons. Anastasia et al. [44] compared hippocampal neurons of Met/Met, Val/Val and Val/Met BDNF knock-in mice and reported that the presence of the Met66 polymorphism induced a structural change in the pro-peptide domain of proBDNF. They observed that only the Met66-, but not Val66- pro-peptide induced acute growth cone retraction through a differential engagement to a Sortilin receptor, implying that such a structural change in the pro-domain region of pro-BDNF may infer increased bioactivity.

Petoukhov and colleagues [45] demonstrated that Progranulin, a growth factor that regulates lysosome function with impact ranging from trophic factor support for neurons to suppression of microglial activation, also co-localizes and is co-transported with BDNF in hippocampal neuron synapses in both axons and dendrites. They indicated that the two proteins are transported within neurons in either an anterograde or retrograde manner, depending upon synaptic activity recruitment. Subsequently, Eckenstaler et al. [46] using live-cell imaging demonstrated the role of calcium-dependent activator protein 1 in the maturation and exocytosis of BDNF- containing DCVs. Besides regulating the intragranular $\mathrm{pH}$ in BDNFcontaining secretory granules, calcium-dependent activator protein was observed to be involved in the regulation of fusion-competent secretory granules and BDNF release in dendrites, following exocytosis. Aravamudan et al. [47], comparing control versus inflammatory conditions, reported that BDNF secretion can be regulated at multiple levels in human smooth muscle cells. Although BDNF release by these cells is probably slower than in highly regulated synaptic vesicular exocytosis such as in neurons, it was demonstrated that local levels of extracellular BDNF are regulated by the inflammatory response, providing the basis for an autocrine role of BDNF in inflammatory states.

More recently, Uegaki and colleagues [48] using plasmid constructs expressing recombinant human BDNF and surface plasmon resonance reflectivity measurements demonstrated that the pro-peptide domain of pro-BDNF binds with high affinity to the mature BDNF domain, and that this interaction is enhanced at acidic compared to neutral $\mathrm{pH}$ conditions. Their findings implied that such binding is more stable in intracellular compartments as trafficking vesicles than in the extracellular milieu. They have also observed that the Val66Met polymorphism had a stabilizing effect on the heterodimeric complex of pro-BDNF, enhancing Met66 pro-BDNF stability in both acidic and neutral $\mathrm{pH}$ conditions, in comparison to the Val66 pro-BDNF. 


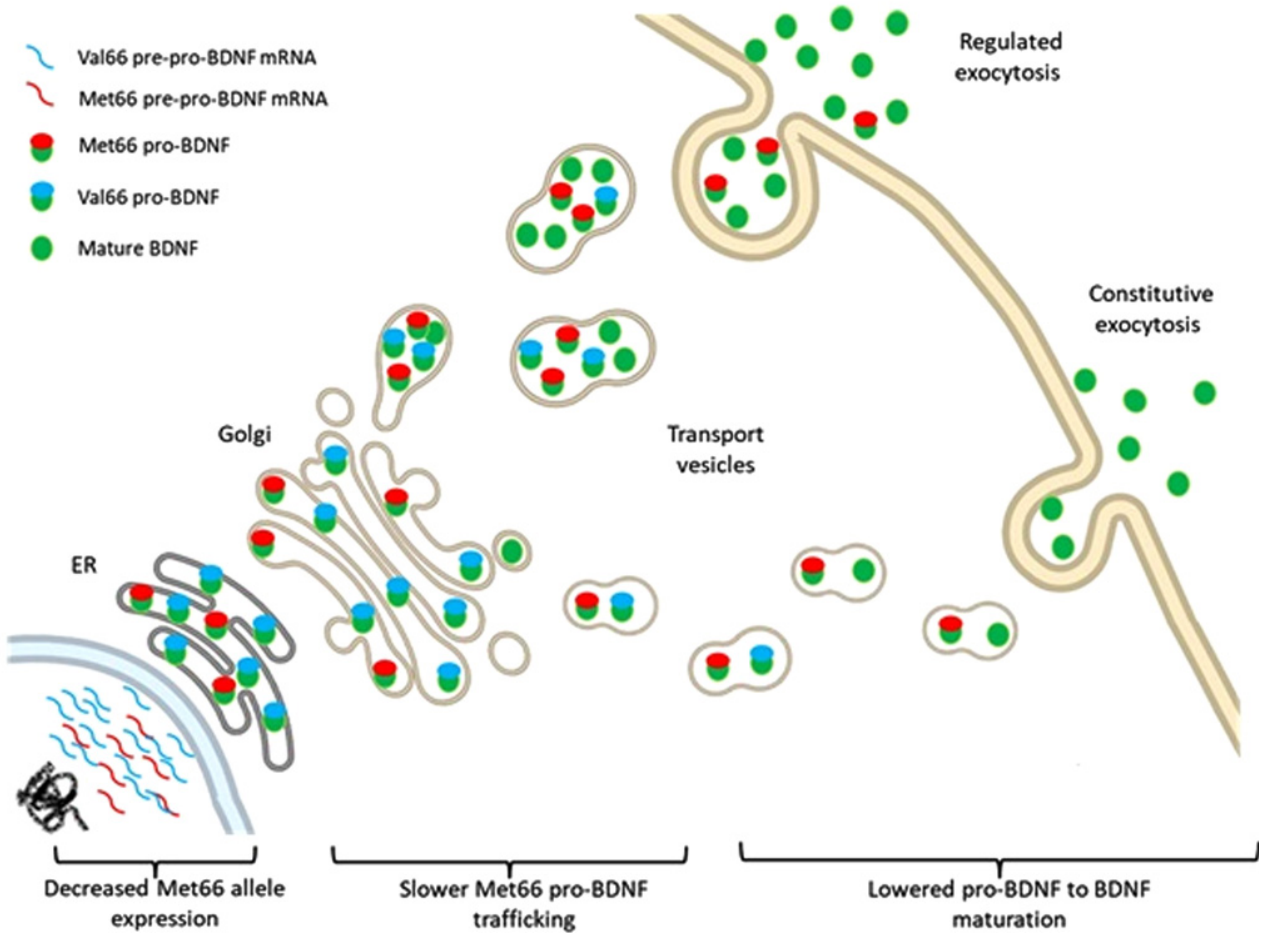

Fig. 2. Schematic illustration for the Val66- and Met66- $B D N F$ alleles expression, post translational modifications and release.

\section{DISCUSSION}

Knowledge on the molecular mechanisms involving $B D N F$ expression and protein formation has experienced considerably growth in the past few decades. In line with the findings on $B D N F$ expression, the current experimental science on the subcellular processes involving BDNF synthesis indicates that the presence of the Val66Met polymorphism may also alter BDNF function at the secretory level.

As the most abundant neurotrophin produced in the adult brain, BDNF is essential to the maintenance of neuroplasticity and the preservation of cognition (i.e., memory and the executive functions). Each of these processes requires a fine control of the timing and quantity of BDNF production, which relies to a certain extent on the pro-BDNF cleavage into BDNF [49]. Experimental models have demonstrated that the Val66Met presence directly affects the sorting and cleavage of the pro-BDNF isoform into the secretory pathways [35, 37, 43, 48]. This results in a more concentrated distribution of both pro-BDNF and BDNF in the neuronal cell bodies, than in dendrites and axons. The Met66 presence also appears to negatively influence the activity-dependent release of the mature BDNF form from DCVs in synaptic terminals upon depolarization.

The importance of the post-translational processing of pro-BDNF for the functioning of BDNF as a trophic factor, such as sorting and enzymatic cleavage, has been consistently confirmed by the studies reviewed. The release system and signaling pathways of BDNF are key regulators of neuronal activity and synaptic strength, while the activity-dependent release of BDNF (regulated secretory pathway) is important for synaptic communication and plasticity [50]. The constitutive release of BDNF exerts a tonic modulation of the connection between neurons, and neurons to non-neuronal tissue expressing TrkB [51, 52]. In neurons, the TGN is the main regulator of these systems and localizes in the cell nucleus, dendrites, and in some segments of the axon, allowing for precise control of membrane composition [53]. The pro-BDNF molecules are thus packaged in the TGN and relocated to sites of release in the axon and dendrites to be recruited as BDNF during synaptic activity, by calcium-mediated exocytosis [45, 54].

The pro-peptide sequence of translated pro-BDNF, however, potentially influences the protein's transport 
and maturation from the ER through the TGN until the secretory vesicles, by altering the protein folding and/or transport signaling [55]. In the case of $B D N F$ Val66Met polymorphism, it appears that the rate of pro-BDNF folding and transportation within the ER-TNG system affects BDNF localization and availability in the secretory vesicles throughout the neuronal membrane [56]. A rigorous intrinsic control of the intracellular ratios of pro-BDNF/BDNF is thus imperative for proper brain function. The mature BDNF molecule is the predominant isoform located in the DCVs at the neuronal membrane [40, 41, 43]. However, before reaching the membrane, a gradual shift from the pro-BDNF to BDNF molecule is seen during the maturation process of the secretory granule [39].

Data shows that $B D N F$ transcripts are translated into a pro-BDNF peptide that can be sorted either into constitutive or regulated secretory systems [32-35]. This provides a differential and independent regulation of BDNF into separate membrane compartments on the neuron's surface $[57,58]$. However, abnormal trafficking and secretion of BDNF is found in the presence of a $B N D F$ Val66Met polymorphism, which has been detected by several investigators [36, 37, 42]. Impaired sorting and distribution of BDNF into the vesicles of the regulated secretory pathway on the synaptic sites of neurons shed light on the importance of a critically stable expression/production of BDNF (see more at de Assis et al., 2021). A stabilizing effect is seen when the Met66 allele is present in the pro-BDNF molecule, which results in a less effective cleavage observed along the BDNF maturation throughout TGN trafficking, leading to an slight increase in pro-BDNF secretion relative to BDNF [48]. Moreover, Anastasia et al. [44] postulated that there is a functional change in the pro domain of the pro-BDNF molecule containing the Val66Met polymorphism that affects its stability that is reflected on BDNF production.

Once pro-BDNF is released in the extracellular matrix and/or the circulation, protease systems support the cleavage of pro-BDNF into BDNF. For instance, tissue plasminogen activator (tPA) rapidly generates BDNF from pro-BDNF by converting plasminogen into plasmin [59-63]. It is well stablished that any dysfunction in the $B D N F$ gene affects the development and maintenance of normal brain function [64]. The impact of the Met66 presence might be sufficient to alter the fine-tuning of synapticregulated neuroplasticity processes, not necessarily affecting released BDNF concentrations. In addition, the pro-apoptotic signaling of pro-BDNF, eventually released by the regulated secretory pathway, via p75NTR might yet be buffered by extracellular systems of cleavage, whereas there is no available data on whether or how the Met66 substitution affects the constitutive release of BDNF and pro-BDNF.

During nervous system development, the release and interaction of BDNF with TrkB receptors initiates several pathway cascades that regulate local transcription of proteins in neural precursor cells to promote cell proliferation and differentiation [65, 66]. During this process, the pro-BDNF molecule helps define the strong neuronal connections by depressing weak connections and neuronal growth processes through p75NTR signaling interaction [67-69]. Consequently, pro-BDNF is not expected to be released in any significant amounts in the adult brain.

It was observed throughout the studies that the $B D N F$ Val66Met polymorphism may alter BDNF function by each diminishing gene expression levels and/or impairing pro-BDNF conversion into BDNF, potentially changing the gradient released pro-BDNF/BDNF ratios from cells [32, 34-38, 42]. While BDNF signaling is putative for the nervous system formation and maintenance, a rigorous control of pro-BDNF release should be critical for the formation of neuronal circuits in the healthy adult brain. Thus, the studies examined in this review suggest that a slight alteration in the gradient of BDNF isoforms released by cells, as a result of the Val66Met polymorphism interference, might have a negative impact on brain function and might be considered a relevant factor for the development of neuro-psychiatric disorders.

\section{FUNDING}

No funding was obtained for this study.

\section{CONFLICTS OF INTEREST/COMPETING INTERESTS}

We have no conflict of interest to declare.

AVAILABILITY OF DATA AND MATERIAL

Not applicable.

\section{CODE AVAILABILITY}

Not applicable. 


\section{AUTHORS' CONTRIBUTIONS}

Conceptualization, G.G.A.; Systematic Search, G.G.A. and J.R.H.; Results analysis and article writing, G.G.A. and J.R.H.

\section{ADDITIONAL DECLARATIONS FOR ARTICLES IN LIFE SCIENCE JOURNALS THAT REPORT THE RESULTS OF STUDIES INVOLVING HUMANS AND/OR ANIMALS}

Not applicable.

\section{ETHICS APPROVAL (INCLUDE APPROPRIATE APPROVALS OR WAIVERS)}

Not applicable.

\section{CONSENT TO PARTICIPATE (INCLUDE APPROPRIATE STATEMENTS)}

Not applicable.

\section{CONSENT FOR PUBLICATION}

Not applicable.

\section{REFERENCES}

[1] Rafieva M, L. V, Gasanov E. Neurotrophin Propeptides: Biological Functions and Molecular Mechanisms. Curr Protein Pept Sci. 2015; 17:298-305.

[2] Song M, Martinowich K, Lee FS. BDNF at the synapse: Why location matters. Molecular Psychiatry. 2017;22: 1370-5.

[3] Bondarenko EA, et al. Genetic analysis of BDNF, GNB3, MTHFR, ACE and APOE variants in major and recurrent depressive disorders in Russia. Int J Med Sci. 2016;13: 977-83.

[4] O'Connor DT, et al. Early alteration in glomerular reserve in humans at genetic risk of essential hypertension: Mechanisms and consequences. Hypertension. 2001;37:898-906.

[5] Li L, et al. BDNF and NGF signals originating from sensory ganglia promote cranial motor axon growth. Exp Brain Res. 2020;238:111-9.

[6] Park H, Poo MM. Neurotrophin regulation of neural circuit development and function. Nat Rev Neurosci. 2013;14: 7-23.

[7] Notaras M, van den Buuse M. Brain-Derived Neurotrophic Factor (BDNF): Novel Insights into Regulation and Genetic Variation. Neuroscientist. 2019;25:434-54.

[8] Brigadski T, Leßmann V. The physiology of regulated BDNF release. Cell and Tissue Research. vol. 382 (Cell and Tissue Research, 2020).
[9] Kojima M, Ishii C, Sano Y, Mizui T, Furuichi T. Journey of brain-derived neurotrophic factor: from intracellular trafficking to secretion. Cell Tissue Res. 2020;382:125-34.

[10] Zhou P, et al. Polarized Signaling Endosomes Coordinate BDNF-Induced Chemotaxis of Cerebellar Precursors. Neuron. 2007;55:53-68.

[11] Evans SF, et al. Neuronal brain-derived neurotrophic factor is synthesized in excess, with levels regulated by sortilinmediated trafficking and lysosomal degradation. J Biol Chem. 2011;286:29556-67.

[12] Gibon J, et al. proBDNF and p75NTR control excitability and persistent firing of cortical pyramidal neurons. J Neurosci. 2015;35:9741-53.

[13] Teng HK, et al. ProBDNF induces neuronal apoptosis via activation of a receptor complex of p75NTR and sortilin. J Neurosci. 2005;25:5455-63.

[14] Bothwell M, Recent advances in understanding contextdependent mechanisms controlling neurotrophin signaling and function [version 1; peer review: 3 approved]. F1000Research. 2019;8:1658.

[15] Maisonpierre PC, et al. Human and rat brain-derived neurotrophic factor and neurotrophin-3: Gene structures, distributions, and chromosomal localizations. Genomics. 1991;10:558-68.

[16] Hunt SE, et al. Ensembl variation resources. Database (Oxford). 2018, (2018).

[17] Neves-Pereira M, et al. BDNF gene is a risk factor for schizophrenia in a Scottish population. Mol Psychiatry. 2005; 10:208-12.

[18] Kocabas NA, et al. Brain-derived neurotrophic factor gene polymorphisms: Influence on treatment response phenotypes of major depressive disorder. Int Clin Psychopharmacol. 2011;26:1-10.

[19] Domschke $\mathrm{K}$, et al. Brain-derived neurotrophic factor (BDNF) gene: No major impact on antidepressant treatment response. Int J Neuropsychopharmacol. 2010;13: 93-101.

[20] Czarnowicki T, et al. Effect of short-term liver X receptor activation on epidermal barrier features in mild to moderate atopic dermatitis: A randomized controlled trial. Ann Allergy, Asthma Immunol. 2018;120:631-640.e11.

[21] de Assis GG, Gasanov EV. BDNF and Cortisol integrative system - Plasticity vs. degeneration: Implications of the Val66Met polymorphism. Frontiers in Neuroendocrinology. 2019;55:100784.

[22] Vinberg M, et al. The BDNF Val66Met polymorphism: Relation to familiar risk of affective disorder, BDNF levels and salivary cortisol. Psychoneuroendocrinology. 2009;34:1380-9.

[23] Shimizu E, Hashimoto K, Iyo M. Ethnic difference of the BDNF 196G/A (val66met) polymorphism frequencies: The possibility to explain ethnic mental traits. Am J Med Genet. 2004;126B:122-3.

[24] De Assis GG, et al. The Val66 and Met66 Alleles-Specific Expression of BDNF in Human Muscle and Their Metabolic Responsivity. Front Mol Neurosci. 2021;14:1-12.

[25] Lemos JR, et al. Peripheral vascular reactivity and serum BDNF responses to aerobic training are impaired by the BDNF Val66Met polymorphism. Physiol. Genomics. 2016; 48:116-23.

[26] Froud A, Murphy J, Cribb L, Ng CH, Sarris J. The relationship between dietary quality, serum brain-derived neurotrophic factor (BDNF) level, and the Val66met polymorphism in predicting depression. Nutr Neurosci. 2019; 22:513-21. 
[27] Ryan KM, Dunne R, McLoughlin DM. BDNF plasma levels and genotype in depression and the response to electroconvulsive therapy. Brain Stimul. 2018;11:1123-31.

[28] Yoshimura R, et al. The brain-derived neurotrophic factor (BDNF) polymorphism Val66Met is associated with neither serum BDNF level nor response to selective serotonin reuptake inhibitors in depressed Japanese patients. Prog Neuro-Psychopharmacology Biol Psychiatry. 2011; 35:1022-5

[29] Colzato LS, Van der Does AJW, Kouwenhoven C, Elzinga BM, Hommel B. BDNF Val66Met polymorphism is associated with higher anticipatory cortisol stress response, anxiety, and alcohol consumption in healthy adults. Psychoneuroendocrinology. 2011;36:1562-9.

[30] Colle R, et al. Brain-derived neurotrophic factor Val66Met polymorphism and 6-month antidepressant remission in depressed Caucasian patients. J Affect Disord. 2015;175:233-40.

[31] Felmingham KL, Dobson-Stone C, Schofield PR, Quirk GJ, Bryant RA. The brain-derived neurotrophic factor Val66Met polymorphism predicts response to exposure therapy in posttraumatic stress disorder. Biol Psychiatry. 2013;73:1059-63.

[32] Seidah NG, Benjannet S, Pareek S, Chrétien M, Murphy RA. Cellular processing of the neurotrophin precursors of NT3 and BDNF by the mammalian proprotein convertases. FEBS Lett. 1996;379:247-50.

[33] Heymach JV, Krüttgen A, Suter U, Shooter EM. The regulated secretion and vectorial targeting of neurotrophins in neuroendocrine and epithelial cells. J Biol Chem. 1996;271:25430-7.

[34] Mowla SJ, et al. Differential sorting of nerve growth factor and brain-derived neurotrophic factor in hippocampal neurons. J Neurosci. 1999; 19:2069-80.

[35] Mowla SJ, et al. Biosynthesis and Post-translational Processing of the Precursor to Brain-derived Neurotrophic Factor. J Biol Chem. 2001;276:12660-6.

[36] Egan MF, et al. The BDNF val66met polymorphism affects activity-dependent secretion of BDNF and human memory and hippocampal function. Cell. 2003;112:257-69.

[37] Chen ZY, et al. Variant Brain-Derived Neurotrophic Factor (BDNF) (Met66) Alters the Intracellular Trafficking and Activity-Dependent Secretion of Wild-Type BDNF in Neurosecretory Cells and Cortical Neurons. J Neurosci. 2004;24:4401-11.

[38] Wu YJ, et al. Nerve Growth Factor, Brain-Derived Neurotrophic Factor, and Neurotrophin-3 Are Sorted to Dense-Core Vesicles and Released Via the Regulated Pathway in Primary Rat Cortical Neurons. J Neurosci Res. 2004;75:825-34.

[39] Wang LC, Meijer HK, Humbel BM, Jenks BG, Roubos EW. Activity-dependent dynamics of coexisting brainderived neurotrophic factor, pro-opiomelanocortin and $\alpha$-melanophore-stimulating hormone in melanotrope cells of Xenopus laevis. J Neuroendocrinol. 2004;16:19-25.

[40] $\mathrm{Li} \mathrm{H}$, et al. Sorting of vesicular monoamine transporter 2 to the regulated secretory pathway confers the somatodendritic exocytosis of monoamines. Neuron. 2005;48:619-33.

[41] Salio C, Averill S, Priestley JV, Merighi A. Costorage of BDNF and neuropeptides within individual dense-core vesicles in central and peripheral neurons. Dev Neurobiol. 2007;67:326-38.

[42] Jiang X, Zhou J, Mash DC, Marini AM, Lipsky RH. Human BDNF isoforms are differentially expressed in cocaine addicts and are sorted to the regulated secretory pathway independent of the Met66 substitution. NeuroMolecular Med. 2009;11:1-12.

[43] Dieni S, et al. BDNF and its pro-peptide are stored in presynaptic dense core vesicles in brain neurons. J Cell Biol. 2012;196:775-88.

[44] Anastasia A, et al. Val66Met polymorphism of BDNF alters prodomain structure to induce neuronal growth cone retraction. Nat Commun. 2013;4:1-12.

[45] Petoukhov E, et al. Activity-dependent secretion of progranulin from synapses. J Cell Sci. 2013;126:5412-21.

[46] Eckenstaler R, Lessmann V, Brigadski T. CAPS1 effects on intragranular $\mathrm{pH}$ and regulation of BDNF release from secretory granules in hippocampal neurons. J Cell Sci. 2016;129:1378-90.

[47] Aravamudan B, Thompson MA, Pabelick CM, Prakash YS. Mechanisms of BDNF regulation in asthmatic airway smooth muscle. Am J Physiol - Lung Cell Mol Physiol. 2016;311:270-9.

[48] Uegaki K, et al. BDNF binds its pro-peptide with high affinity and the common val66met polymorphism attenuates the interaction. Int J Mol Sci. 2017;18:1042.

[49] Lu B, Nagappan G, Lu Y. BDNF and synaptic plasticity, cognitive function, and dysfunction. Handb Exp Pharmacol. 2015;220:223-50.

[50] Sasi M, Vignoli B, Canossa M, Blum R. Neurobiology of local and intercellular BDNF signaling. Pflugers Arch. 2017;469:593-610.

[51] Feng N, et al. Constitutive BDNF/TrkB signaling is required for normal cardiac contraction and relaxation. Auton Neurosci. 2015;192:51-2.

[52] Cunha C, Brambilla R, Thomas KL. A simple role for BDNF in learning and memory? Front Mol Neurosci. 2010;3.

[53] Kienzle C, von Blume J, Secretory cargo sorting at the transGolgi network. Trends Cell Biol. 2014;24:584-93.

[54] Berg EA, et al. Isolation and characterization of substance P-containing dense core vesicles from rabbit optic nerve and termini. J Neurosci Res. 2000;62:830-9.

[55] Dannies PS. Protein hormone storage in secretory granules: Mechanisms for concentration and sorting. Endocrine Reviews vol. 20 https://academic.oup.com/edrv/articleabstract/20/1/3/2530802 (1999).

[56] Horton AC, Ehlers MD. Dual modes of endoplasmic reticulum-to-Golgi transport in dendrites revealed by livecell imaging. J Neurosci. 2003;23:6188-99.

[57] González C, Cornejo VH, Couve A. Golgi bypass for local delivery of axonal proteins, fact or fiction? Current Opinion in Cell Biology. 2018;53:9-14.

[58] Sytnyk V, Leshchyns'ka I, Dityatev A, Schachner M. Trans-Golgi network delivery of synaptic proteins in synaptogenesis. J Cell Sci. 2004;117:381-8.

[59] Kwinter DM, Lo K, Mafi P, Silverman MA. Dynactin regulates bidirectional transport of dense-core vesicles in the axon and dendrites of cultured hippocampal neurons. Neuroscience. 2009;162:1001-10.

[60] Lee R, Kermani P, Teng KK, Hempstead BL. Regulation of cell survival by secreted proneurotrophins. Science 2001;(80-.). 294:1945-8.

[61] Lochner JE, et al. Efficient copackaging and cotransport yields postsynaptic colocalization of neuromodulators associated with synaptic plasticity. Dev Neurobiol. 2008; 68:1243-56.

[62] Angelucci F, Čechová K, Průša R, Hort J. Amyloid beta soluble forms and plasminogen activation system in Alzheimer's disease: Consequences on extracellular maturation of brain-derived neurotrophic factor and therapeutic 
implications. CNS Neuroscience and Therapeutics. 2019; 25:303-13.

[63] Rodier M, et al. Exogenous t-PA administration increases hippocampal mature BDNF levels. Plasmin- Or NMDAdependent mechanism? PLoS One. 2014;9:92416.

[64] Cattaneo A, Cattane N, Begni V, Pariante CM, Riva MA. The human BDNF gene: peripheral gene expression and protein levels as biomarkers for psychiatric disorders. Translational Psychiatry. 2016;6:e958.

[65] Bartkowska K, Paquin A, Gauthier AS, Kaplan DR, Miller FD. Trk signaling regulates neural precursor cell proliferation and differentiation during cortical development. Development. 2007;134:4369-80.
[66] Leal G, Comprido D, Duarte CB. BDNF-induced local protein synthesis and synaptic plasticity. Neuropharmacology. 2014;76:639-56.

[67] Gonzalez A, Moya-Alvarado G, Gonzalez-Billaut C, Bronfman FC. Cellular and molecular mechanisms regulating neuronal growth by brain-derived neurotrophic factor. Cytoskeleton. 2016;73:612-28.

[68] Panja D, Bramham CR. BDNF mechanisms in late LTP formation: A synthesis and breakdown. Neuropharmacology. 2014;76:664-76.

[69] Hempstead BL. Brain-Derived Neurotrophic Factor: Three Ligands, Many Actions. Trans Am Clin Climatol Assoc. 2015;126:9-19. 